\title{
Explicit Fourth-Derivative Two-Step Linear Multistep Method for Ordinary Differential Equations
}

JOURNAL OF RESEARCH

\section{Wusu Ashiribo Senapon and Akanbi Moses Adebowale}

Department of Mathematics, Lagos State University, Ojo. Lagos, Nigeria.

\section{Correspondence}

Wusu Ashiribo Senapon, Department of Mathematics, Lagos State University, Ojo. Lagos, Nigeria.

Email:wussy_ash@yahoo.com

\section{Funding information}

This research work is self sponsored.

\section{Abstract:}

Introduction: Many problems from science and engineering are modeled by Ordinary Differential Equations (ODEs) whose solutions describe the temporal evolution of the modeled processes. In most cases however, the arising equations are too complex to be solved analytically. Consequently, their solutions have to be approximated by numerical methods.

Aims: In this article, we propose an Explicit Fourth-Derivative two-step Linear Multistep Method (FD2LMM) for systems of first-order ordinary differential equations.

Materials and Methods: The proposed method is constructed by using the maximal order criteria which is obtained through the associated linear difference operator. The starting values used by the proposed method are obtained by suitable single-step method.

Results: The order, consistency, linear stability, and the convergence properties of the method are discussed. Numerical experiments are performed and the results are compared with those of existing methods in the literature. Conclusion: Explicit fourth-derivative two-step linear multistep method (FD2LMM) for ordinary differential equation was constructed using the order criteria and the associated linear difference operator. The stability property of the constructed method was also analysed. The results obtained from the numerical examples show that accurate and efficient compared with methods in the literature.

Keywords: Fourth-derivative, Linear Multistep Method, Initial Value Problem, System of equations, Stability

All co-authors agreed to have their names listed as authors.

This is an open access article under the terms of the Creative Commons Attribution License, which permits use, distribution and reproduction in any medium, provided the original work is properly cited.

(c) 2018 The Authors. Journal of Research and Reviews in Science - JRRS, A Publication of Lagos State University 


\section{INTRODUCTION}

In this article, the first-order initial value problem in ordinary differential equations

$$
u^{\prime}=f(t, u), \quad t \in\left[t_{0}, T\right], u\left(t_{0}\right)=\eta_{0}
$$

where $f: \mathbb{R} \times \mathbb{R}^{n} \mapsto \mathbb{R}^{n}, u, \eta_{0} \in \mathbb{R}^{n}$ and $t_{0}, T \in \mathbb{R}$ satisfies the Lipschitz condition [1], is considered.

Several of the techniques for solving (1) are generally based on the Taylor algorithm and are usually of the Runge-Kutta type or classical linear multistep type [2, 1, 3, 4]. The Taylor algorithm is one of the earliest known scheme for solving (1). In recent time, several modifications are being to the classical Runge-Kutta and linear multistep method which result in different families of methods. Some of such methods that are obtained by a modification (the use of averages other than the arithmetic average) of the classical Runge-Kutta method are the methods proposed by the authors in [5, 6, 7]. However, the quest for methods with improved accuracy has been on the increase since the work of the author in [8]. Based on the idea of an early work, the author in [8] attempted using Multiderivative Linear Multistep Method (MLMM) with $k=1$ in the second derivative formulae. In the work by the authors in [9], a family of second derivative Adams-type methods (SDAMs) of order up to $2 k+2$ (where $\mathrm{k}$ is the steplength) for initial value problems was proposed. Similarly, the authors in [10, 11] constructed explicit Runge-Kutta type methods involving up to second derivative. In the work of [12], explicit Runge-Kutta schemes of stages up to four with the first derivative were constructed. Following the work [12], a family of Runge-Kutta type method with higher order derivatives up to the second derivative was derived by the author in [13]. Here, we analyze the construction and implementation of explicit fourth-derivative two-step linear multistep method (FD2LMM) for solving (1) taking into account the order criteria and the associated linear difference operator.

\section{MATERIAL AND METHODS}

\subsection{Construction of Method}

The form of the method to be constructed in this work is

$$
\begin{aligned}
u_{n+2}-u_{n}= & \sum_{i=0}^{3} h^{i+1} \sum_{j=0}^{1} \beta_{i j} f_{n+j}^{(i)} \\
= & h\left(\beta_{00} f_{n}+\beta_{01} f_{n+1}\right)+ \\
& h^{2}\left(\beta_{10} f_{n}^{(1)}+\beta_{11} f_{n+1}^{(1)}\right)+ \\
& h^{3}\left(\beta_{20} f_{n}^{(2)}+\beta_{21} f_{n+1}^{(2)}\right)+ \\
& h^{4}\left(\beta_{30} f_{n}^{(3)}+\beta_{31} f_{n+1}^{(3)}\right)
\end{aligned}
$$

The associated linear difference operator, $\mathcal{L}[h, \gamma]$ of (2) is obtained as

$$
\begin{aligned}
\mathcal{L}[h, \gamma] u(t)= & u(t+2 h)-u(t)- \\
& h\left(\beta_{00} u^{(1)}(t)+\beta_{01} u^{(1)}(t+h)\right)- \\
& h^{2}\left(\beta_{10} u^{(2)}(t)+\beta_{11} u^{(2)}(t+h)\right)- \\
& h^{3}\left(\beta_{20} u^{(3)}(t)+\beta_{21} u^{(3)}(t+h)\right)- \\
& h^{4}\left(\beta_{30} u^{(4)}(t)+\beta_{31} u^{(4)}(t+h)\right)
\end{aligned}
$$

where $\gamma:=\left(\beta_{00}, \beta_{01}, \beta_{10}, \beta_{11}, \beta_{20}, \beta_{21}, \beta_{30}, \beta_{31}\right)$. The operator, $\mathcal{L}$ is dimensionless. Applying the operator $\mathcal{L}$ to

$$
t, t^{2}, t^{3}, t^{4}, t^{5}, t^{6}, t^{7}, t^{8}, t^{9}
$$

, the corresponding system of equations for the dimensionless moments

$$
L_{m}^{*}(\gamma):=\left.h^{-m} \mathcal{L}[h, \gamma] t^{m}\right|_{t=0}
$$

is

$$
\begin{aligned}
& L_{1}^{*}(\gamma):=-\beta_{00}-\beta_{01}+2=0 \\
& L_{2}^{*}(\gamma):=-2\left(\beta_{01}+\beta_{10}+\beta_{11}-2\right)=0 \\
& L_{3}^{*}(\gamma):=-3 \beta_{01}-6 \beta_{11}-6 \beta_{20}-6 \beta_{21}+8=0 \\
& L_{4}^{*}(\gamma):=-4\left(\beta_{01}+3 \beta_{11}+6 \beta_{21}+6 \beta_{30}+6 \beta_{31}-4\right)=0 \\
& L_{5}^{*}(\gamma):=-5 \beta_{01}-4\left(5 \beta_{11}+15 \beta_{21}+30 \beta_{31}-8\right)=0 \\
& L_{6}^{*}(\gamma):=-2\left(3 \beta_{01}+15 \beta_{11}+60 \beta_{21}+180 \beta_{31}-32\right)=0 \\
& L_{7}^{*}(\gamma):=-7 \beta_{01}-42 \beta_{11}-210 \beta_{21}-840 \beta_{31}+128=0 \\
& L_{8}^{*}(\gamma):=-8\left(\beta_{01}+7 \beta_{11}+42 \beta_{21}+210 \beta_{31}-32\right)=0 \\
& L_{9}^{*}(\gamma):=-9 \beta_{01}-8\left(9 \beta_{11}+63 \beta_{21}+378 \beta_{31}-64\right)=0 .
\end{aligned}
$$

Examining the above system, we see that the system is compatible for the set

$\left\{L_{1}^{*}(\gamma)=0, L_{2}^{*}(\gamma)=0, \cdots, L_{8}^{*}(\gamma)=0\right\}$. Hence the maximum $M$ for which the system $L_{m}^{*}(\gamma)=0, \quad m=1,2, \cdots M-1$ is compatible is 9 . Now, solving the system $L_{1}^{*}(\gamma)=0, L_{2}^{*}(\gamma)=$ $0, \cdots, L_{8}^{*}(\gamma)=0$ for the coefficients

$$
\beta_{00}, \beta_{01}, \beta_{10}, \beta_{11}, \beta_{20}, \beta_{21}, \beta_{30}, \beta_{31}
$$

results in

$$
\begin{aligned}
& \beta_{00}=34, \\
& \beta_{01}=-32, \\
& \beta_{10}=\frac{110}{7}, \\
& \beta_{11}=\frac{128}{7}, \\
& \beta_{20}=\frac{20}{7}, \\
& \beta_{21}=-\frac{80}{21} \\
& \beta_{30}=\frac{22}{105}, \\
& \beta_{31}=\frac{65}{35}
\end{aligned}
$$


From the result above, the fourth-derivative two-step explicit linear multistep method (FD2LMM) proposed in this work is

$$
\begin{aligned}
u_{n+2}-u_{n}= & h\left(34 f_{n}-32 f_{n+1}\right)+ \\
& \frac{1}{7} h^{2}\left(110 f_{n}^{(1)}+128 f_{n+1}^{(1)}\right)+ \\
& \frac{1}{21} h^{3}\left(60 f_{n}^{(2)}-80 f_{n+1}^{(2)}\right)+ \\
& \frac{1}{105} h^{4}\left(22 f_{n}^{(3)}+48 f_{n+1}^{(3)}\right)
\end{aligned}
$$

\section{RESULTS}

\subsection{Order, Local Truncation Error and Consis- tency of The FD2LMM Method}

\subsubsection{Order of the FD2LMM method}

Lemma 3.1 The FD2LMM method (2), and hence the associated operator $L_{m}^{*}$ defined by (4) have order $\mathrm{p}$ if and only if

$$
L_{r}^{*} \equiv 0, \quad r=0,1, \cdots, p, \quad L_{r+1}^{*} \not \equiv 0 .
$$

Theorem 3.2 The FD2LMM method (6) is of order 8.

\section{Proof:}

Since with the coefficient values (5), $L_{m}^{*}=0$ for $m=0,1, \cdots, 8$ and

$$
L_{9}^{*}:=-9 \beta_{01}-8\left(9 \beta_{11}+63 \beta_{21}+378 \beta_{31}-64\right),
$$

substituting the coefficients (5) into (8) results in $L_{9}^{*}=\frac{736}{35} \neq$ 0 . Hence the FD2LMM method (6) is of order 8.

\subsubsection{Local Truncation Error of the FD2LMM method}

The general expression of the leading term of the local truncation error (Ite) for a method of the form (2) with order $p$ can be written in the form (see [3])

$$
\text { lte }(t)=(-1)^{p+1} h^{p+1} \frac{L_{p+1}^{*}}{(p+1) !} D^{p+1} u(t) .
$$

Since the FD2LMM method (6) is of order $p=8$, the corresponding local truncation error is obtained as

$$
\text { lte }(t)=-h^{9} \frac{736}{35.9 !} u^{(9)}(t) .
$$

\subsubsection{Consistency}

Theorem 3.3 The linear multistep method FD2LMM method (2) is said to be consistent if it has order $p \geq 1$, [3].

Lemma 3.4 The FD2LMM method (6) is consistent since it has order $p=8>1$.

\subsection{Stability Polynomial of The FD2LMM Method}

The first and second characteristics polynomials of the FD2LMM method (6) are respectively given by

$$
\begin{aligned}
\rho(\xi) & =\xi^{2}-1 \\
\sigma_{i}(\xi) & =\sum_{j=0}^{1} \beta_{i j} \xi^{j}, \quad i=0,1,2,3 .
\end{aligned}
$$

From the above, the corresponding stability polynomial is obtained as

$$
\begin{aligned}
\pi(\xi, \bar{h})= & \rho(\xi)-\sum_{i=0}^{3} \bar{h}^{i+1} \sigma_{i}(\xi) \\
= & \frac{1}{105}\left(-48 \bar{h}^{4} \xi-22 \bar{h}^{4}+400 \bar{h}^{3} \xi-300 \bar{h}^{3}-\right. \\
& 1920 \bar{h}^{2} \xi-1650 \bar{h}^{2}+3360 \bar{h} \xi- \\
& \left.3570 \bar{h}+105 \xi^{2}-105\right)
\end{aligned}
$$

\subsection{Numerical Results}

Numerical experiments confirming the expectations regarding our constructed method are presented in this section. Our method was applied to two standard problems: a linear system of dimension 3 and a nonlinear system of dimension 2 . These problems were studied in the literature by the authors in [9], [14] and [15]. The results produced by our method are compared with those produced by the methods (SDAM), (Amodio) and (Wu-Xia) proposed in [9], [14] and [15] respectively. The accuracy and efficiency of our method are demonstrated using these examples.

\subsubsection{Problem 1}

Consider the following initial value problem considered by the authors in [14] on the range $0 \leq t \leq 1$.

$$
\begin{array}{ll}
u_{1}^{\prime}=-21 u_{1}+19 u_{2}-20 u_{3}, & u_{1}(0)=1 \\
u_{2}^{\prime}=19 u_{1}-21 u_{2}+20 u_{3}, & u_{2}(0)=0 \\
u_{3}^{\prime}=40 u_{1}-40 u_{2}-40 u_{3}, & u_{3}(0)=-1 .
\end{array}
$$


The analytical solution of the system is obtained as

$$
\begin{aligned}
& u(t)_{1}=\frac{1}{2} e^{-40 t}\left(e^{38 t}+\sin (40 t)+\cos (40 t)\right) \\
& u(t)_{2}=-\frac{1}{2} e^{-40 t}\left(e^{38 t}-\sin (40 t)-\cos (40 t)\right) \\
& u(t)_{3}=-e^{-40 t}(\cos (40 t)-\sin (40 t)) .
\end{aligned}
$$

The problem was solved using different values of steplength $h$ and the maximum relative error for each step was obtained and compared with those given in [14] and [15]. The comparison is presented in Table 1 and it is clear that our method produces more accurate results than those produced in [14] and [9].

\subsubsection{Problem 2}

The second problem considered in this paper is the nonlinear system from [15] and also studied in [9],

$$
\begin{aligned}
y_{1}^{\prime}(t)=-1002 u_{1}+1000 u_{2} ; & u_{1}(0)=1 \\
y_{2}^{\prime}(t)=u_{2}-u_{2}\left(1+u_{2}\right) ; & u_{2}(0)=1
\end{aligned}
$$

The analytical solution of problem 2 is given as

$$
\begin{aligned}
& u_{1}(t)=\exp (-2 t) \\
& u_{2}(t)=\exp (-t)
\end{aligned}
$$

This problem was solved within the ranges $t \in[0,1]$ and $t \in[0,10]$ with steplengths $h=\{0.008, h=0.006\}$ and $h=$ $\{0.002, h=0.001\}$ respectively. For this problem, we compared the results of our method at $t=1$ and $t=10$ with those obtained by the methods in [9] and [15]. From the results, it is clear that our method gave better results compare with those produced by the method of [15]. Also, our method compete favourably the that of the author in [9]. Details of the comparison are presented in Table 2.

\section{CONCLUSION}

Explicit fourth-derivative two-step linear multistep method (FD2LMM) for ordinary differential equation was constructed using the order criteria and the associated linear difference operator. The stability property of the constructed method was also analysed. The results obtained from the numerical examples show that accurate and efficient compared with methods in the literature.

\section{AUTHORS' CONTRIBUTIONS}

All authors participated actively in this research work and the writing of the manuscript.
CONSENT (WHERE EVER APPLICABLE)

Consent form has been approved by all authors.

\section{REFERENCES}

[1] Henrici P. Discrete Variable Methods in ODEs, John Wiley, New York, 1962.

[2] Dalquist G. G., Numerical integration of ordinary differential equations., Math. Scand. 1956; 4:69-86.

[3] Lambert, J.D., Computational Methods in ODEs, Wiley, New York. 1973.

[4] Butcher, J.C. Numerical Methods for Ordinary Differential Equations. Wiley, 2008.

[5] Akanbi, M.A. On 3-stage Geometric Explicit RungeKutta Method for Singular Autonomous Initial Value Problems in Ordinary Differential Equations, Computing. 2011; 92:243-263.

[6] Wusu A.S, Okunuga S.A and Sofoluwe A.B., A ThirdOrder Harmonic Explicit Runge-Kutta Method for Autonomous Initial Value Problems, Global Journal of Pure and Applied Mathematics. 2012; 8(4): 441-451.

[7] Wusu A.S., Akanbi M.A. and Fatimah B.O. On the Derivation and Implementation of a Four Stage Harmonic Explicit Runge-Kutta Method, Applied Mathematics. 2015; 6:694-699.

[8] Cash J.R., On exponentially fitting of composite multiderivative Linear Methods, SIAM J. Numerical Anal. 1981; 18(5):808-821.

[9] Jator S. N., Sahi, R. K., Boundary value technique for initial value problems based on Adamstype second derivative methods, International Journal of Mathematical Education in Science and Technology, First published on: 07 June 2010 (iFirst)

[10] Akanbi, M.A., Okunuga S. A. On Region of Absolute Stability and Convergence of 3-Stage Multiderivative Explicit Runge-Kutta Methods, Journal of the Sciencea Research and Development Institute. 2006; 10:83-100.

[11] Akanbi, M.A., Okunuga S. A., Sofoluwe A. B. Error Bounds for 2-Stage Multiderivative Explicit Runge-Kutta Methods, Advances in Modelling and Analysis. 2008; 45(2):57-72.

[12] Goeken D., Johnson O., Fifth-Order Runge-Kutta with Higher Order Derivative Approximations, Electronic Journal of Differential Equations. 1999; 2:1-9. 
Table 1: The relative errors of the proposed "FD2LMM" method compared with the methods (SDAM) of [9] and (Amodio) [14] for problem 1

\begin{tabular}{|c|c|c|c|c|c|}
\hline \multirow[b]{3}{*}{ Step } & \multicolumn{5}{|c|}{ Relative Error } \\
\hline & FD2LMM & SDAM & Amodio & SDAM & Amodio \\
\hline & $k=2(p=8)$ & $k=2(p=6)$ & $k=5(p=6)$ & $k=3(p=8)$ & $k=7(p=8)$ \\
\hline 20 & $1.5 \times 10^{-7}$ & $2.9 \times 10^{-3}$ & $5.7 \times 10^{-2}$ & $7.5 \times 10^{-4}$ & $2.9 \times 10^{-2}$ \\
\hline 40 & $1.2 \times 10^{-9}$ & $7.3 \times 10^{-5}$ & $8.7 \times 10^{-3}$ & $1.9 \times 10^{-5}$ & $6.8 \times 10^{-3}$ \\
\hline 80 & $7.2 \times 10^{-12}$ & $1.8 \times 10^{-6}$ & $4.9 \times 10^{-4}$ & $1.4 \times 10^{-7}$ & $7.8 \times 10^{-5}$ \\
\hline 160 & $2.9 \times 10^{-15}$ & $3.3 \times 10^{-8}$ & $1.2 \times 10^{-5}$ & $6.4 \times 10^{-10}$ & $4.7 \times 10^{-7}$ \\
\hline 320 & $2.3 \times 10^{-15}$ & $5.1 \times 10^{-10}$ & $2.2 \times 10^{-7}$ & $2.5 \times 10^{-12}$ & $2.3 \times 10^{-9}$ \\
\hline 640 & $1.1 \times 10^{-16}$ & $7.7 \times 10^{-12}$ & $3.7 \times 10^{-9}$ & $9.8 \times 10^{-15}$ & $1.3 \times 10^{-11}$ \\
\hline
\end{tabular}

Table 2: The absolute error of our "FD2LMM" method compared with the "Wu-Xia" method at $t=1$ and $t=10$ on problem 2

\begin{tabular}{|c|c|c|c|c|c|}
\hline \multirow[b]{3}{*}{$\mathrm{t}$} & \multirow[b]{3}{*}{$\mathrm{h}$} & \multicolumn{4}{|c|}{ Absolute Error } \\
\hline & & \multicolumn{2}{|c|}{ Wu-Xia } & \multicolumn{2}{|c|}{ FD2LMM } \\
\hline & & $u_{1}(t)$ & $u_{2}(t)$ & $u_{1}(t)$ & $u_{2}(t)$ \\
\hline 1 & 0.002 & $2.5606 \times 10^{-07}$ & $8.0150 \times 10^{-08}$ & $8.3267 \times 10^{-17}$ & $4.4409 \times 10^{-16}$ \\
\hline 10 & 0.001 & $5.5468 \times 10^{-16}$ & $6.0936 \times 10^{-12}$ & $2.7756 \times 10^{-17}$ & $2.7756 \times 10^{-16}$ \\
\hline
\end{tabular}

Table 3: The absolute error of our "FD2LMM" method compared with the "SDAM" method at $t=1$ and $t=10$ on problem 2

\begin{tabular}{|c|c|c|c|c|c|}
\hline \multirow[b]{3}{*}{$\mathrm{t}$} & \multirow[b]{3}{*}{$\mathrm{h}$} & \multicolumn{4}{|c|}{ Absolute Error } \\
\hline & & \multicolumn{2}{|c|}{ SDAM } & \multicolumn{2}{|c|}{ FD2LMM } \\
\hline & & $u_{1}(t)$ & $u_{2}(t)$ & $u_{1}(t)$ & $u_{2}(t)$ \\
\hline 1 & 0.008 & $1.6348 \times 10^{-14}$ & $0.0000 \times 10^{00}$ & $1.1102 \times 10^{-16}$ & $0.0000 \times 10^{00}$ \\
\hline 10 & 0.006 & $2.4815 \times 10^{-24}$ & $2.0329 \times 10^{-20}$ & $4.1359 \times 10^{-24}$ & $4.0658 \times 10^{-20}$ \\
\hline
\end{tabular}

[13] Wusu A.S. and Akanbi M.A., A Three-Stage Multiderivative Explicit Runge-Kutta Method, American Journal of Computational Mathematics. 2013; 3:121-126.

[14] Amodio P. and Mazzia F., Boundary value methods based on Adams, Appl. Numer. Math. 1995; 18:23-35.

[15] Wu X. and J. Xia, Two low accuracy methods for stiff systems, Appl. Math. Comput.2001; 123:141-153. 